\title{
Syndrome of perimenopausal melatonin deficiency is a new marker of women reproductive system aging
}

\author{
Alexandra Mamontova ${ }^{1}$, Elena Usoltseva $^{2}$, and Andrey Soloviev ${ }^{3}$ \\ ${ }^{1}$ State Budgetary Health Instution "Regional Clinical Hospital N 3", Ministry of Health of the Russian \\ Federation, Russia \\ ${ }^{2}$ Federal State Budgetary Educational Institution of Higher Education "South-Ural State Medical \\ University" of the Ministry of Healthcare of the Russian Federation University, 454092, Vorovskogo \\ Str., 64, Chelyabinsk, Russia \\ ${ }^{3}$ Federal State Budgetary Educational Institution of Higher Education "Northern State Medical \\ University" of the Ministry of Healthcare of the Russian Federation University, 163000, Troitskiy \\ Ave., 51, Arkhangelsk, Russia
}

\begin{abstract}
The aim of our study was to determine a complex of risk factors of the perimenopausal melatonin deficiency syndrome (SPDM) in postmenopausal women and women during menopausal transition suffering from climacteric syndrome. The study involved 278 women. They were divided into two groups: I group included 246 menopausal women and II group included 32 women during the menopausal transition. The Medical History was collected, the menopausal index, and anthropometric measures were determined. The study was conducted with a help of the test questionnaire for melatonin deficiency (TQMD), the women's health questionnaire (WHQ), and detection of the level of 6sulfatoxymelatonin (6-SOMT) in urine. According to the obtained data, risk factors of SPDM in women during the menopausal transition are the chronological age increment, overweight, and the reduction of the estradiol level. The leading risk factors of SPDM in postmenopausal women are the chronological age increment, overweight with visceral type of obesity and changes in the hormonal profile. Thus, SPDM is a new clinical and laboratory marker of aging of the female reproductive system, an important screening tool for detecting the development of $\mathrm{CC}$ in women during the menopausal transition and menopausal women.
\end{abstract}

\section{Introduction}

Female aging is associated with not only a deficiency of sex steroids, reduction of level of the pineal gland hormone - melatonin (MT) also plays an important role in this process. Nowadays, a new concept - perimenopausal melatonin deficiency syndrome (SPDM) is suggested. SPDM is a complex of symptoms, which is often formed amid decrease of the

\footnotetext{
* Corresponding author: Alexandramamontova@rambler.ru

** Corresponding author: Elena-usoltseva@yandex.ru

*** Corresponding author: ASoloviev1@yandex.ru
} 
melatonin synthesis and clinically characterized by the prevalence of complaints of sleep disorders, bodily pain, depression, anxiety/fears and somatic symptoms (fatigue, dizziness, nausea; back and limb pain; tingling in the hands/feet) [1]. SPDM is a donosological state and it is a predecessor of a clinical form of the pathological climacteria development climacteric syndrome (CC). In women during menopausal transition and in postmenopause, it is necessary to make a timely identification of a prenosological state, predictor of CCSPDM. A screening tool, a test questionnaire for melatonin deficiency (TQMD) [2] is used for this purpose. In addition, the 6-SOMT level of urine is measured, and severity of symptoms according to the scales of the Women's Health Questionnaire - a "disease specific" instrument - are evaluated [3].

The aim of our study was to determine a complex of risk factors of SPDM in postmenopausal women and women during the menopausal transition suffering from CC.

\section{Materials and methods}

The study involved 278 women. They were divided into two groups: I group included 246 menopausal women, IIgroup included 32 women during the menopausal transition. The Medical History was collected, modified menopausal index (MMI) and anthropometric measures (Waist-To-Hip-Ratio (WTH), body mass index (BMI), weight gain in recent years) were determined. Bohmann vaginal health index (VHI) was determined as well. The VHI is a system used to evaluate vulvovaginal atrophy on a scale of 1 to 5 . However, 1 point conforms to the highest degree of atrophy, and 5 points conform to a normal state of vaginal mucous. Women filled out TQMD and special instrument-WHQ. According to TQMD, the more scoring of the test is, the more prominent is the MT deficiency. WHQ is a 37-item questionnaire combined into nine scales (the closer the indicator is to zero, the better is the QOL of a woman). We did not evaluate the domain "menstrual symptoms" in postmenopausal women. The level of melatonin metabolite 6-SOMT was determined in evening (at 23.00), night (from 02.00) and morning (at 06.00) urine samples. Serum concentration of luteinizing hormone (LH), follicle-stimulating hormone (FSH), estradiol, prolactin, and testosterone were determined by EIA method. Spearman rank correlation analysis of TQMD, 6-SOMT level and scales of WHQ vs clinical data was used for identification of risk factors of SPDM.

\section{Results and discussion}

\section{TQMD}

Correlation analysis between TQMD and clinical data in the I group shows moderate positive correlation between points of TQMD and weight gain in recent years $(p=0.001)$, moderate positive correlation between points of TQMD and MMI was revealed $(p \leq 0,001)$.

A moderate positive correlation between TQMD and MMI $(p=0.012)$, LH level $(p=$ 0.05 ) and a moderate negative correlation between TQMD and serum testosterone concentration $(\mathrm{p}=0.05)$ were found in the II group. The results are shown in Table 1 and Table 2.

\section{6-SOMT in morning urine samples}

A moderate negative correlation between 6-SOMT in morning urine samples and chronological age $(\mathrm{p} \leq 0,001)$, a weak negative correlation between 6 -SOMT and age at menopause $(p=0.013)$, BMI $(p=0.002)$, MMI were revealed $(p=0.001)$ in the I group. In 
women in the II group only a moderate negative correlation between 6-SOMT in morning urine samples and BMI $(\mathrm{p}=0.026)$ was found. The results are shown in Table 1 and Table 2.

\section{6-SOMT in evening and night urine samples}

A weak negative correlation between the 6-SOMT level in evening urine samples and weight gain in recent years $(\mathrm{p}=0.05)$, a negative moderate correlation between 6-SOMT level at night and MMI $(\mathrm{p}=0.011)$, and strong positive correlation between night concentration of 6-SOMT and VHI $(\mathrm{p}=0.039)$ were indentified in the I group.

The same analysis in the II group revealed, no correlation between the 6-SOMT in evening urine samples and clinical data. Moreover, a moderate positive correlation between 6-SOMT at night and MMI $(\mathrm{p}=0.014)$ and a moderate negative correlation between 6SOMT in night urine samples and FSH $(\mathrm{p}=0.05)$ were found. The results are shown in Table 1 and Table 2.

\section{Women is Health Questionnaire}

In our study, we analyzed the correlation relationships between each scale and clinical data.

\section{Depression (DEP)}

The analysis of correlation relationship between the Dep scale and clinical data revealed a moderate positive correlation with MMI $(\mathrm{p} \leq 0,001)$, and a weak negative correlation with the estradiol level $(\mathrm{p}=0.037)$ in the I group.

In the II group, a moderate positive correlation between Dep scale and MMI was found $(\mathrm{p} \leq 0,001)$.

\section{Somatic symptoms (SOM)}

Several positive correlations were found between the SOM scale, clinical data in the I group of women: a weak correlation with BMI $(p=0.018)$, a moderate correlation with MMI $(p \leq 0,001)$ and with the testosterone level $(p \leq 0,001)$ and one negative moderate correlation with VHI $(p \leq 0,001)$.

The same analysis in the II group of women showed a positive moderate correlation between SOM and chronological age $(\mathrm{p}=0.038)$, BMI $(\mathrm{p}=0.039)$ and MMI $(\mathrm{p} \leq 0,001)$.

\section{Memory/concentration (MEM)}

A weak positive correlation between points of the MEM scale and MMI $(p \leq 0,001)$ was revealed in the I group, and a moderate positive correlation between the MEM scale and BMI $(\mathrm{p}=0.021)$, MMI $(\mathrm{p}=0.05)$ were found in the II group.

\section{Vasomotor symptoms (VAS)}

There were a weak positive correlation between the VAS scale and BMI $(p=0.004)$, weight gain in recent years $(p=0.048)$ and a moderate positive correlation between the VAS scale and the testosterone level $(\mathrm{p}=0.005)$ in the I group of women. In addition, there was a moderate positive correlation between points of the VAS scale and WHT ( $p=0.003)$ in the II group. 


\section{Anxiety/fears (ANX)}

The rank correlation analysis between clinical data and the ANX scale revealed a positive correlation between the ANX scale and MMI in both groups of respondents, however, in the I group the relationship was weak $(p \leq 0,001)$, and in the II - moderate $(p=0.005)$.

\section{Sexual problems (SEX)}

A weak negative correlation between the SEX scale and age at menopause $(p=0.037)$ was found in the I group and a strong negative correlation between the SEX scale and the testosterone level $(\mathrm{p}=0.032)$ was found in the II group.

\section{Sleep (SLE)}

In the I group of women, the following correlations between the SLE scale and clinical data were determined: weak positive correlation relationship with age at menopause $(p=0.038)$, a moderate positive with MMI $(p \leq 0,001)$, and a moderate negative with VHI $(p=0.002)$. No correlation relationship between the SLE scale and clinical data was found in the II group of women.

\section{Menstrual symptoms (MEN)}

The rank correlation analysis between the MEN scale and clinical and data of participants of the II group revealed a moderate positive correlation between the MEN scale and MMI $(\mathrm{p}=0.006)$.

\section{Attractiveness (ATT)}

A weak negative correlation between the scale of ATT and MMI $(\mathrm{p} \leq 0,001)$, a moderate positive correlation between the ATT scale and VHI $(\mathrm{p}=0.028)$ were indentified in the I group. Moreover, there was a moderate negative correlation between the ATT scale and MMI $(\mathrm{p}=0.001)$ in the II group.

It is known that the level of MT is unstable during menstrual cycle and decreases at menopause $[5,6]$. Nowadays a new term is proposed - SPDM, which is formed because of MT synthesis decrease in perimenopausal women [1]. This study shows that CC is formed in women, both at menopausal transition and at menopause affected by SPDM. At the same time, the more profound SPDM, the more severe CC occurs, which emphasizes the important role of SPDM for the prognosis of pathological climacteria.

By now, Russian [8] and foreign [6, 7,9] scientists have published a number of works in which MT is used as a therapeutic for the treatment of patients with CC. Thus, N. Parandavar et all noted the positive effect of MT on psychological, somatic, vasomotor symptoms and reduction of sexual dysfunction in women [6]. In the study of W. Y. Chen et all, the frequency and severity of vasomotor symptoms remained unchanged during treatment with MT, however, there was a significant improvement in the quality of sleep [7]. In our opinion, the positive effects of MT in the treatment of CC are primarily associated with the correction of SPDM, which is formed in these patients even before the appearance of symptoms of pathological climacteria and accompanies CC development.

Within the framework of STRAW +10 , chronological age does not serve as a criterion for determining the stages of aging of the female reproductive system; indicators of $\mathrm{FSH}$, estradiol, anti-Muller hormone, and inhibin B are serve as laboratory criteria [4]. At the same time, MT is one of the hormones that play a key role in the aging of the female body. 
Epiphysis hormone is a powerful antioxidant, and is involved in mechanisms that regulate ovarian aging [6].Moreover, MT can affect the psychoemotional manifestations of CC through the synthesis of gamma-aminobutyric acid (GABA), the maximum concentration of which is at night, when the synthesis of MT increases [6]. With the background of SPDM, metabolic disorders are formed. We have found a correlation between an increase of BMI and the level of 6-COMT, changes in body mass and indicators of TQMD in women during menopausal transition. The findings are consistent with other studies. Thus, A. Goyal et all noted the positive effects of MT in the treatment of metabolic syndrome [10]; other researchers came to similar conclusions [11].

In our study, we have found a moderate inverse correlation between VHI and SOM, SLE scales. In the available literature, we did not find publications that evaluated the effect of MT on the state of the genital mucosa or studied the role of MT in the formation of menopausal genitourinary syndrome. A number of authors have noted the positive effect of MT on sexual function in postmenopausal women [12, 13], scientists associate a similar effect with the effect of MT on the serotonergic system [13]. However, from our point of view, the decrease in VHI indicators, which means, the aggravation of vulvovaginal atrophy, should be considered symptomatic of SPDM.

Table 1. Results of Spearman rank correlation analysis between the test questionnaire of melatonin deficiency, 6-SOMT levels and clinical data, hormonal profile in postmenopausal women.

\begin{tabular}{|c|c|c|c|c|c|}
\hline & & $\begin{array}{l}\text { 6-SOMT in } \\
\text { evening urine } \\
\text { sample }\end{array}$ & $\begin{array}{l}\text { 6-SOMT in } \\
\text { night urine } \\
\text { sample }\end{array}$ & $\begin{array}{l}\text { 6-SOMT in } \\
\text { morning urine } \\
\text { sample }\end{array}$ & TQMD \\
\hline \multirow{3}{*}{ Chronological age } & Spearman R & $-0,168$ & $-0,221$ & $-0,334$ & 0,032 \\
\hline & $\mathrm{P}$ & 0,065 & 0,140 & $<0,001$ & 0,731 \\
\hline & $\mathrm{N}$ & 121 & 46 & 125 & 117 \\
\hline \multirow{3}{*}{ Menopausal age } & Spearman R & $-0,031$ & 0,000 & $-0,222$ & $-0,005$ \\
\hline & $P$ & 0,738 & 0,997 & $\mathbf{0 , 0 1 3}$ & 0,960 \\
\hline & $\mathrm{N}$ & 121 & 46 & 125 & 117 \\
\hline \multirow{3}{*}{ BMI } & Spearman R & $-0,098$ & $-0,235$ & $-0,270$ & 0,149 \\
\hline & $\mathrm{P}$ & 0,285 & 0,116 & 0,002 & 0,109 \\
\hline & $\mathrm{N}$ & 121 & 46 & 125 & 117 \\
\hline \multirow{3}{*}{ WTH } & Spearman R & $-0,035$ & $-0,026$ & $-0,083$ & 0,074 \\
\hline & $\mathrm{P}$ & 0,730 & 0,869 & 0,399 & 0,467 \\
\hline & $\mathrm{N}$ & 102 & 44 & 106 & 98 \\
\hline \multirow{3}{*}{ MMI } & Spearman R & $-0,130$ & $-0,373$ & $-0,281$ & 0,530 \\
\hline & $\mathrm{P}$ & 0,156 & 0,011 & 0,001 & $<0,001$ \\
\hline & $\mathrm{N}$ & 121 & 46 & 125 & 117 \\
\hline \multirow{3}{*}{ FSH } & Spearman R & 0,000 & $-0,065$ & 0,078 & $-0,043$ \\
\hline & $\mathrm{P}$ & 0,998 & 0,719 & 0,506 & 0,743 \\
\hline & $\mathrm{N}$ & 70 & 33 & 74 & 60 \\
\hline
\end{tabular}




\begin{tabular}{|c|c|c|c|c|c|}
\hline \multirow{3}{*}{ LH } & Spearman R & $-0,078$ & 0,017 & $-0,030$ & 0,146 \\
\hline & $\mathrm{P}$ & 0,549 & 0,936 & 0,811 & 0,301 \\
\hline & $\mathrm{N}$ & 62 & 25 & 66 & 52 \\
\hline \multirow{3}{*}{ Estradiol } & Spearman R & $-0,107$ & 0,081 & $-0,091$ & $-0,046$ \\
\hline & $\mathrm{P}$ & 0,418 & 0,708 & 0,474 & 0,752 \\
\hline & $\mathrm{N}$ & 60 & 24 & 64 & 49 \\
\hline \multirow{3}{*}{ Prolactine } & Spearman R & 0,045 & 0,036 & $-0,189$ & 0,186 \\
\hline & $\mathrm{P}$ & 0,781 & 0,887 & 0,219 & 0,227 \\
\hline & $\mathrm{N}$ & 40 & 18 & 44 & 44 \\
\hline \multirow{3}{*}{ Testosterone } & Spearman R & 0,141 & 0,389 & $-0,109$ & $-0,035$ \\
\hline & $\mathrm{P}$ & 0,385 & 0,090 & 0,483 & 0,824 \\
\hline & $\mathrm{N}$ & 40 & 20 & 44 & 43 \\
\hline \multirow{3}{*}{ VHI } & Spearman R & $-0,285$ & 0,833 & $-0,065$ & $-0,084$ \\
\hline & $\mathrm{P}$ & 0,178 & 0,039 & 0,746 & 0,794 \\
\hline & $\begin{array}{c}\mathrm{N} \text { - почему } \\
\text { так мало? }\end{array}$ & 24 & 6 & 27 & 12 \\
\hline \multirow{3}{*}{$\begin{array}{l}\text { Weight gain in } \\
\text { recent years }\end{array}$} & Spearman R & $-0,185$ & $-0,126$ & $-0,011$ & 0,308 \\
\hline & $\mathrm{P}$ & 0,051 & 0,427 & 0,907 & 0,001 \\
\hline & $\mathrm{N}$ & 112 & 42 & 116 & 112 \\
\hline
\end{tabular}

TQMD - The test questionnaire of melatonin deficiency, WTH- Waist-To-Hip-Ratio, BMI- Body mass index, LH-Luteinizing hormone, FSH- Follicle-stimulating hormone,VHI- Vaginal health index,6-SOMT- 6-sulfatoxymelatonin in urine.

Table 2. Results of Spearman rank correlation analysis between the test questionnaire of melatonin deficiency, 6-SOMT levels and clinical data, hormonal profile in women during menopausal transition

\begin{tabular}{|c|c|c|c|c|c|}
\hline \multicolumn{2}{|c|}{} & $\begin{array}{c}\text { 6-SOMT in } \\
\text { evening urine } \\
\text { sample }\end{array}$ & $\begin{array}{c}\text { 6-SOMT in } \\
\text { night urine } \\
\text { sample }\end{array}$ & $\begin{array}{c}\text { 6-SOMT in } \\
\text { morning urine } \\
\text { sample }\end{array}$ & TQMD \\
\hline \multirow{5}{*}{ Chronological age } & $\begin{array}{c}\text { Spearman } \\
\mathrm{R}\end{array}$ & $-0,065$ & 0,150 & $-0,020$ & $-0,084$ \\
\cline { 2 - 7 } & $\mathrm{P}$ & 0,723 & 0,625 & 0,914 & 0,610 \\
\cline { 2 - 7 } & $\mathrm{N}$ & 32 & 13 & 32 & 39 \\
\hline \multirow{3}{*}{ BMI } & $\begin{array}{c}\text { Spearman } \\
\mathrm{R}\end{array}$ & $-0,005$ & 0,275 & $-0,394$ & 0,383 \\
\cline { 2 - 7 } & $\mathrm{P}$ & 0,977 & 0,364 & $\mathbf{0 , 0 2 6}$ & $\mathbf{0 , 0 1 7}$ \\
\cline { 2 - 7 } & $\mathrm{N}$ & 32 & 13 & 32 & 38 \\
\hline \multirow{3}{*}{ WTH } & $\begin{array}{c}\text { Spearman } \\
\mathrm{R}\end{array}$ & 0,114 & $-0,100$ & $-0,278$ & 0,323 \\
\cline { 2 - 7 } & $\mathrm{P}$ & 0,555 & 0,769 & 0,144 & 0,067 \\
\cline { 2 - 7 } & $\mathrm{N}$ & 29 & 11 & 29 & 33 \\
\hline
\end{tabular}




\begin{tabular}{|c|c|c|c|c|c|}
\hline \multirow{3}{*}{ MMI } & $\begin{array}{c}\text { Spearman } \\
\mathrm{R} \\
\end{array}$ & 0,057 & 0,660 & $-0,226$ & 0,398 \\
\hline & $\mathrm{P}$ & 0,755 & 0,014 & 0,214 & 0,012 \\
\hline & $\mathrm{N}$ & 32 & 13 & 32 & 39 \\
\hline \multirow{3}{*}{ FSH } & $\begin{array}{c}\text { Spearman } \\
\mathrm{R}\end{array}$ & $-0,095$ & $-0,667$ & 0,095 & 0,213 \\
\hline & $\mathrm{P}$ & 0,667 & 0,050 & 0,665 & 0,328 \\
\hline & $\mathrm{N}$ & 23 & 9 & 23 & 23 \\
\hline \multirow{3}{*}{ LH } & $\begin{array}{c}\text { Spearman } \\
\text { R }\end{array}$ & 0,068 & $-0,429$ & 0,232 & 0,443 \\
\hline & $\mathrm{P}$ & 0,777 & 0,337 & 0,325 & 0,050 \\
\hline & $\mathrm{N}$ & 20 & 7 & 20 & 20 \\
\hline \multirow{3}{*}{ Estradiol } & $\begin{array}{c}\text { Spearman } \\
\text { R }\end{array}$ & 0,106 & $-0,357$ & $-0,095$ & $-0,349$ \\
\hline & $\mathrm{P}$ & 0,686 & 0,432 & 0,717 & 0,170 \\
\hline & $\mathrm{N}$ & 17 & 7 & 17 & 17 \\
\hline \multirow{3}{*}{ Prolactine } & $\begin{array}{c}\text { Spearman } \\
\mathrm{R}\end{array}$ & 0,196 & 0,200 & $-0,133$ & $-0,194$ \\
\hline & $\mathrm{P}$ & 0,542 & 0,747 & 0,681 & 0,546 \\
\hline & $\mathrm{N}$ & 12 & 5 & 12 & 12 \\
\hline \multirow{3}{*}{ Testosterone } & $\begin{array}{c}\text { Spearman } \\
\text { R }\end{array}$ & 0,126 & $-0,174$ & $-0,161$ & $-0,576$ \\
\hline & $\mathrm{P}$ & 0,696 & 0,742 & 0,617 & 0,050 \\
\hline & $\mathrm{N}$ & 12 & 6 & 12 & 12 \\
\hline \multirow{3}{*}{ VHI } & $\begin{array}{c}\text { Spearman } \\
\text { R }\end{array}$ & & . & . & . \\
\hline & $P$ & . & . & . & . \\
\hline & $\mathrm{N}$ & 0 & 0 & 0 & 0 \\
\hline \multirow{3}{*}{$\begin{array}{l}\text { weight gain in } \\
\text { recent years }\end{array}$} & $\begin{array}{c}\text { Spearman } \\
\mathrm{R} \\
\end{array}$ & $-0,239$ & 0,131 & $-0,334$ & 0,276 \\
\hline & $\mathrm{P}$ & 0,211 & 0,685 & 0,076 & 0,115 \\
\hline & $\mathrm{N}$ & 29 & 12 & 29 & 34 \\
\hline
\end{tabular}

TQMD - The test questionnaire of melatonin deficiency, WTH- Waist-To-Hip-Ratio, BMI- Body mass index, LH-Luteinizing hormone, FSH- Follicle-stimulating hormone,VHI- Vaginal health index,6-SOMT- 6-sulfatoxymelatonin in urine.

Table 3. Results of Spearman rank correlation analysis of WHQ questionnaire scales and modified menopausal index in postmenopausal women and women during the menopausal transition.

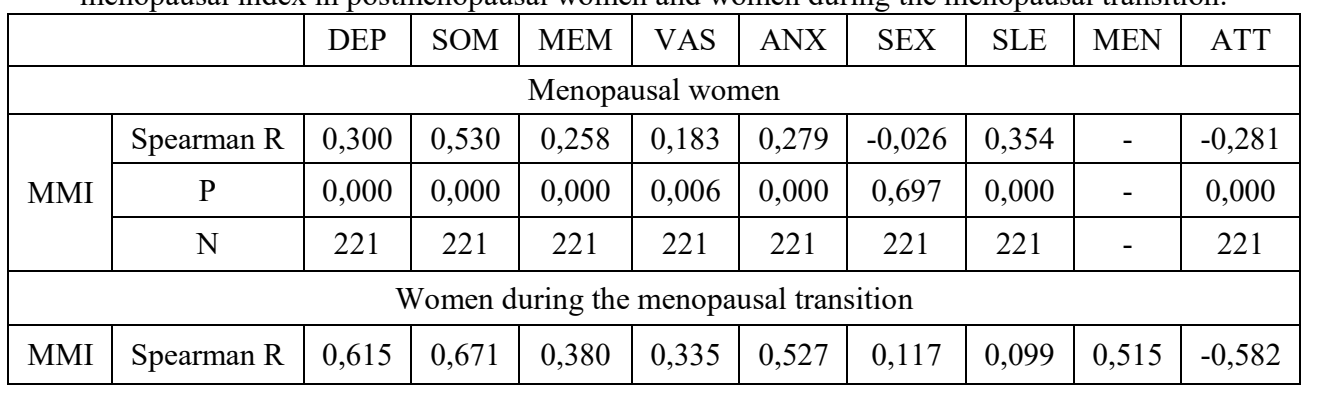




\begin{tabular}{|c|c|c|c|c|c|c|c|c|c|c|} 
& $\mathrm{P}$ & 0,001 & 0,000 & 0,051 & 0,088 & 0,005 & 0,563 & 0,623 & 0,006 & 0,001 \\
\cline { 2 - 10 } & $\mathrm{N}$ & 27 & 27 & 27 & 27 & 27 & 27 & 27 & 27 & 27 \\
\hline
\end{tabular}

MMI-Modified menopausal index, DEP- Depression, SOM- Somatic symptoms, MEM-

Memory/concentration, VAS- Vasomotor symptoms, ANX- Anxiety/fears, SEX- Sexual problems, SLE- Sleep, MEN- Menstrual symptoms, ATT- Attractiveness.

\section{Conclusion}

Thus, our study revealed correlations between each criteria of SPDM and MMI (Table 3), VHI. So, every woman suffering from $\mathrm{CC}$ should be identified as a patient with comorbid pathology: CC and SPDM. The reduction of VHI, increment of presentation of vulvovaginal atrophy, should be considered as one of the markers of the SPDM presence.

A profound SPDM complies with severity of CC in menopausal transition women and postmenopausal women. According to the obtained data, risk factors of SPDM in women in menopausal transition are the chronological age increment, overweight, and the reduction of the estradiol level. The leading risk factors of SPDM in postmenopausal women are the chronological age increment, overweight with visceral type of obesity and changes in hormonal profile of patients - increase of the LH, FSH levels and decrease of the testosterone level. It is important to notice, that the correlation relationship of FSH and the level of 6-COMT in nighturine samples indicates the lack of peak of the MT synthesis in menopausal transition women and postmenopausal women. That is a pathognomonic symptom of a significant reduction of the epiphysis function.

Thus, SPDM is a new clinical and laboratory marker of aging of the female reproductive system, an important screening tool for detecting the development of CC in women in the menopausal transition and menopausal women. We found an important natural connection: the more profound SPDM associates with the more severe CC. Each patient suffering from CC should be studied from the point of view of comorbid pathology SPDM. From the point of view of predicting and personifying modern medicine, the inclusion of MT in the treatment regimens of CC is justified, this will increase not only the effectiveness of complex therapy, but also the QOL of women, and therefore, stimulate the participation of medical care to this group of patients.

\section{References}

1. E.N. Usoltseva, A.G. Soloviev, A.G. Mamontova, Advancts in Gerontology, 32(4), 516-523 (2019)

2. C. Dalle, The Guide, Global Aging Management, S.l, 1, 496 (2010)

3. M.S. Hunter, Health Qual Life Outcome, 1, 41 (2003)

4. Aging of the reproductive system: new criteria STRAW +1 , Women's health, $\mathbf{5}(\mathbf{8 1})$ (2013)

5. A.Yarci Gursoy, M. Kiseli, G.S. Caglar, Climacteric, 18, 1-7 (2015)

6. N. Parandavar, K. Abdali, S. Keshtgar, M. Emamghoreishi, S. Amooee, Iran Journal Public Health, 43(10), 1405-1416 (2014)

7. W.Y. Chen, A. Giobbie-Hurder, K. Gantman, J. Savoie, R. Scheib, L.M. Parker, E.S. Schernhammer, Breast Cancer Res. Treat., 145(2), 381-388 (2014)

8. I.V. Kuzneczova, D.I. Burchakov, Ginekologiya, 17(5), 44-48 (2015)

9. M.P. Kotlarczyk, H.C. Lassila, C.K. O'Neil, F.D. Amico, L.T. Enderby, P.A. WittEnderby, J.L. Balk, Journal of Pineal Research, 52(4), 414-26 (2012) 
10. A. Goyal, P. Terry, H.M. Superak, C.L. Nell-Dybdahl, R. Chowdhury, L.S. Phillips, M. Kutner, Diabetology \& Metabolic Syndrome, 6(124) (2014)

11. M. Koziróg, A.R. Poliwczak, P. Duchnowicz, M. Koter-Michalak, J. Sikora, M. Broncel, Journal of Pineal Research, 50, 261-266 (2011)

12. N. Parandavar, K. Abdali, S. Keshtgara, M. Emamghoreishi, S. Amooeeh, L. Mosalanejad, Iran Journal Public Health, 6(4), 149-155 (2017)

13. N. Parandavar, A. Khadijeh, S. Keshtga, M. Emamghoreishi, S. Amooee, Iran Journal Public Health, 43(10), 1405-16 (2014) 